2

3

4

\title{
THE METABOLIC ENGINE OF ENDOTHELIAL CELLS
}

2

3

9

10

\author{
Kim D. Falkenberg ${ }^{1,2}$, Katerina Rohlenova $^{1,2}$, Yonglun $_{L^{3}}{ }^{3-6}$ \& Peter Carmeliet $^{1,2}$
}
(1) Laboratory of Angiogenesis and Vascular Metabolism, Department of Oncology, KU Leu- ven, Leuven, B-3000, Belgium; (2) Laboratory of Angiogenesis and Vascular Metabolism, Center for Cancer Biology, VIB, Leuven, B-3000, Belgium; (3) Department of Biomedicine, Aarhus Uni- versity, Aarhus DK-8000, Denmark; (4) Lars Bolund Institute of Regenerative Medicine, BGI- Qingdao, Qingdao 266555, China; (5) BGI-Shenzhen, Shenzhen 518083, China; (6) China Na- tional GeneBank, BGI-Shenzhen, Shenzhen 518120, China.

Running title: Endothelial cell metabolism

Keywords: Endothelial cells, metabolism, angiogenesis

\section{Editorial correspondence:}

\section{P. Carmeliet, M.D., Ph.D.}

Laboratory of Angiogenesis and Vascular Metabolism

VIB, KU Leuven

Center for Cancer Biology

Campus Gasthuisberg O\&N4

Herestraat 49 - 912,

B-3000, Leuven, Belgium

tel: 32-16-37.32.02; fax: 32-16-37.25.85

e-mail: peter.carmeliet@kuleuven.vib.be 


\section{ABSTRACT}

27 Endothelial cells (ECs) line the quiescent vasculature, but can form new blood vessels (a process 28 termed angiogenesis) in disease. Strategies targeting angiogenic growth factors have been clinically 29 developed for the treatment of malignant and ocular diseases. Studies over the past decade docu30 mented that several pathways of central carbon metabolism are necessary for EC homeostasis and 31 growth, and that strategies that stimulate or block EC metabolism can be used to respectively pro32 mote or inhibit vessel growth. In this review, we provide an updated oversight of our growing under33 standing of central carbon metabolic pathways in ECs and the therapeutic opportunities of targeting 34 EC metabolism.

35

36

37 


\section{INTRODUCTION}

39 Blood vessels supply oxygen and nutrients, drain waste, regulate vascular patency, produce angi40 ocrine signals for tissue regeneration and serve as gateways for immune cell trafficking ${ }^{1}$. They are

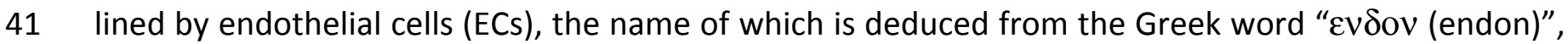
42 meaning "inside", referring to their inner location in the blood vessel. While ECs are quiescent in 43 healthy adults (except in a few conditions such as pregnancy, etc.), they become rapidly activated in 44 response to signals, produced by tissues in need of vascularization, which results in the formation of new blood vessels - a process referred to as angiogenesis ${ }^{1,2}$. When activated, ECs undergo a phenotypic switch ${ }^{3}$ and differentiate into proliferating stalk cells to elongate the sprout and into migratory tip cells to guide the sprout at the forefront, a position that is adopted by the fittest cell via dynamic competition (Fig. 1) ${ }^{4}$.

Dysfunction of ECs has been implicated in the pathophysiology of diabetes ${ }^{5}$ and atherosclerosis $^{6}$, and contributes to the development of peripheral artery disease ${ }^{7}$, coronary heart disease and myocardial infarction ${ }^{8,9}$, cerebrovascular disease ${ }^{10}$, as well as kidney disease ${ }^{11}$. Excessive angiogenesis, on the other hand, promotes diseases such as cancer, blinding eye disease and pulmonary arterial hypertension $(\mathrm{PAH})^{12-14}$.

Traditional anti-angiogenic therapies (AATs) target EC-activating growth factor pathways, such as VEGF signaling. However, while clinically used for cancer and blinding eye disease treatment, these approaches lack sufficient efficacy and treated patients develop resistance - partly due to the com-

57 pensatory effect of other signaling pathways that activate ECs ${ }^{1,15}$. In search of new therapeutic opportunities that may bypass these limitations, a new concept, proposed in 2009, postulated that ECS rely on metabolic adaptations to execute the instructions of angiogenic signals to form new blood vessels (Fig. 1) ${ }^{16}$. In this review, we provide an update of our current understanding of how ECs utilize 
61 metabolism, often in a particular manner, and discuss possible therapeutic opportunities of targeting

62 EC metabolism for the development of alternative therapeutic strategies to promote or inhibit vessel

63 growth. Rather than providing a historical, all-encompassing overview, we highlight principles by illus-

64 trating key examples.

65

66

67

68

69

70

71

72

73

74

75

76

77

78

79

80

81

82

\section{Endothelial Metabolism}

\section{Glycolysis AND Side PATHWAYS}

Blood vessels deliver oxygen to nearly all tissues in the body, yet ECs are not fully taking profit of the access to oxygen in the blood and generate most of their ATP anaerobically via glycolysis (Fig. 2,3 ) - in contrast to many other cell types ${ }^{17}$. This is striking, as the conversion of glucose to pyruvate during glycolysis yields only 2 moles of ATP per mole of glucose, making it a less efficient means for ATP production than oxidative phosphorylation (OXPHOS), yielding 36 moles of ATP. However, this - at first sight - apparent contra-intuitive behavior offers advantages. Indeed, reliance on anaerobic metabolism lowers the production of reactive oxygen species (ROS) and enables ECs to vascularize regions deprived of oxygen and nutrients, which would otherwise be impossible if their energy production was primarily dependent on oxidative glucose metabolism. Consistently, in glucose-replete conditions, ECs are relatively resistant to hypoxia, but they become sensitive to oxygen depletion when glucose levels drop ${ }^{18}$. Another advantage is that ECs, by consuming little oxygen, can transfer larger amounts of oxygen to perivascular cells, a primary objective of why ECs developed during evolution.

While glycolytic flux is high in all types of ECs, it is lower in quiescent ECs due to downregulation of glycolytic gene expression by the transcription factors FOXO1 and Notch ${ }^{19,20}$. In contrast, growth factors like VEGF upregulate glycolytic gene expression in angiogenic ECs, and both proliferating stalk cells and migratory tip cells rely on glycolysis ${ }^{17,21}$. In tip cells, glycolytic energy production 
83 not only takes place in the cytosol, but also in local "ATP hot spots" in lamellipodia and filopodia, 84 which facilitate energy-intensive actin cytoskeletal remodeling and promote tip cell competitiveness $85 \quad 17$.

Hyperproliferative ECs in cancer and PAH have high rates of glycolysis and employ this pathway not only for energy production but also for biomass synthesis ${ }^{22,23}$. In fact, ECs are hyperglycolytic compared to many other cell types ${ }^{17}$. Hence, the upregulated glycolysis represents an attractive metabolic target for manipulating pathological angiogenesis in these diseases ${ }^{24}$. Subject of intense research is, for instance, the role of the glycolysis stimulator 6-Phosphofructo-2-Kinase/Fructose-2,6Biphosphatase 3 (PFKFB3, Fig. 2), a VEGFR target ${ }^{25}$ that is upregulated in tumor ECs (TECS) ${ }^{22}$. Inhibiting or silencing PFKFB3 reduces the growth and migration of ECs in vitro, rendering them more quiescent ${ }^{17,25,26}$. Of relevance, pharmacological blockade of PFKFB3 in mouse tumor models normalizes abnormal tumor vessels by tightening vascular barrier integrity and improving vessel maturation ${ }^{27}$. Extending these observations, overexpression of PFKFB3 in turn enhances glycolysis, which promotes vessel sprouting ${ }^{17}$. Indeed, mosaic overexpression of PFKFB3 in an EC spheroid sprouting model in vitro or in a transgenic zebrafish angiogenesis model in vivo boosts tip cell behavior, which provided the first evidence that a change in EC metabolism by itself, even without a change in angiogenic signaling, was capable of altering vessel sprouting ${ }^{17}$, showcasing the important role of EC metabolism.

Further illustrating the reliance of angiogenic ECs on glycolysis, inhibition of other glycolytic proteins, like hexokinase 2 (Fig. 2) ${ }^{21}$, which catalyzes an initial step in glycolysis, and adenosine recep102 tor $\mathrm{A} 2 \mathrm{a}^{28}$, an upstream activator of glycolysis, similarly impairs angiogenesis. Surprisingly, the anti103 angiogenic effect observed by inhibiting another glycolytic enzyme, PKM2, is mechanistically based on altered NFkB signaling instead of glycolytic attenuation or impairment ${ }^{29}$. In glucose-limiting conditions, such as during vessel sprouting into hypoglycemic tissues, angiogenic ECs rely on utilization of 
106 glucose stored in internal glycogen reservoirs, as impairment of glycogen breakdown by inhibiting gly107 cogen phosphorylase (GP) reduces EC viability and impairs EC migration ${ }^{30}$.

108 Intermediates of the glycolytic pathway are utilized as substrates for side pathways, like the 109 pentose phosphate pathway (PPP) (Fig. 2). The PPP is involved in nucleotide biosynthesis and provides 110 NADPH for fatty acid synthesis, redox homeostasis and the synthesis of nitric oxide (NO), a key regula111 tor of vascular tone, by endothelial nitric oxide (NO) synthase (eNOS) ${ }^{31}$. Indeed, increasing the PPP 112 flux reduces ROS accumulation in ECs exposed to high glucose levels ${ }^{32}$. Hyperproliferative ECs in PAH 113 feature an elevated PPP flux, presumably in order to meet the cells' demand for nucleotides during 114 DNA replication (Fig. 3) ${ }^{33}$. In line, inhibition of the PPP by silencing rate-limiting enzymes in oxidative 115 or non-oxidative side arms (respectively, glucose-6-phosphate dehydrogenase (G6PDH) ${ }^{32}$ or trans116 ketolase (TKT) ${ }^{30}$, Fig. 2) reduces proliferation of angiogenic ECs. Considering that inhibiting the glyco117 Iytic flux in angiogenic ECs not only impedes energy production but also PPP-dependent biomass syn118 thesis ${ }^{22}$, glycolysis manipulation may represent a therapeutic approach for targeting hyper-activated 119 ECs (Fig. 3A).

120 Another glycolytic side pathway implicated in angiogenesis is the hexosamine biosynthesis 121 pathway (HBP, Fig. 2). The HBP produces UDP-GIcNAc (uridine diphosphate N-acetylglucosamine) in122 ducing protein glycosylation, which regulates the activity of target proteins, like for example VEGFR2 $123 \quad 3^{34}$. In ECs, HBP-mediated protein glycosylation might have an inhibitory effect on angiogenesis, since 124 blocking glycosylation by silencing the HBP key enzyme glutamine:fructose-6-phosphate amidotrans125 ferase 1 (GFAT1) ${ }^{35}$ induces vascular sprouting, whereas boosting glycosylation has opposite effects ${ }^{36}$. 126 The synthesis of serine, another pathway utilizing glycolytic intermediates, will be discussed below.

\section{TCA CyCle and MitochondRial Oxidative Phosphorylation}


128 A fraction of glycolysis-derived carbons enters the tricarboxylic acid (TCA) cycle in the form of acetyl129 CoA or oxaloacetate (Fig. 2) ${ }^{17,37}$. ECs are particular in how they sustain the TCA cycle, as a substantial 130 amount of acetyl-CoA entering the TCA cycle is derived from fatty acids through fatty acid oxidation 131 (FAO, see below) in conjunction with other anaplerotic substrates likely derived from glucose and/or 132 amino acids ${ }^{37}$. The TCA cycle is vital for biomass production in ECs, as its intermediates serve as sub133 strates for various anabolic pathways, such as biosynthesis of nucleotides or amino acids like aspar134 tate ${ }^{37-39}$. Also, the TCA cycle regenerates the reducing agent NADH for OXPHOS. Mitochondrial vol135 ume in ECs typically (with an exception of brain ECs) represents only $2-5 \%$ of the cytoplasmic volume, 136 in contrast, for instance, to the $32 \%$ volume density of mitochondria in oxidative cardiac muscle cells 13740,41 . Thus, it is not surprising that mitochondria and OXPHOS were traditionally considered to have 138 only marginal roles in ECs. However, recent reports documenting that OXPHOS is crucial for EC func139 tion urge to reconsider the role of mitochondria in ECs ${ }^{42-46}$.

Mitochondrial respiration serves various purposes, including ATP production by utilizing re141 ducing agents (Fig. 3). The respiratory machinery consists of multi-protein complexes that transfer 142 electrons derived from $\mathrm{NADH}$ and $\mathrm{FADH}_{2}$ while pumping protons across the inner mitochondrial 143 membrane (complexes I-IV). The consequent proton gradient is used by the ATP synthase to generate 144 ATP. Blockade of OXPHOS complexes I and III (by using piericidin A and antimycin A, respectively, or 145 by inactivating ubiquinol-cytochrome C reductase complex III subunit VII (UQCRQ)) compromises EC 146 proliferation and impairs developmental and pathological angiogenesis ${ }^{47}$. In line with these findings, 147 indirect interference with OXPHOS - due to heme synthesis defects, which impedes the activity of 148 heme-containing complexes III and IV (see Serine Metabolism) ${ }^{48}$, or by oxaloacetate accumulation as 149 a result of depletion of oxaloacetate decarboxylase FAHD1, which inhibits complex II activity ${ }^{49}-$ also 150 causes proliferation defects in ECs. 
152 by complex I, which diminishes the TCA cycle flux, lowers aspartate levels and impairs oxidative pro153 cesses requiring $\mathrm{NAD}^{+}$(Fig. 3) ${ }^{47,50}$. Regenerating $\mathrm{NAD}^{+}$levels by ectopic expression of electron 154 transport chain-independent NADH oxidase rescues aspartate levels and proliferation in ECs. There155 fore, a key function of OXPHOS in ECs is to maintain the NAD ${ }^{+} / \mathrm{NADH}$ ratio in order to sustain biosyn156 thetic processes during proliferation, such as aspartate production ${ }^{47}$. This is in accordance with the 157 fact that, as mentioned above, ECs cover the major part (up to 85\%) of their energy demand by gly158 colysis instead of utilizing OXPHOS (Fig. 3), indicating a negligible role of mitochondrial respiration in 159 ATP production in glucose-rich conditions ${ }^{17}$. Mitochondrial respiration in quiescent ECs (displaying 160 lower proliferation and anabolic needs) is downregulated by FOXO1 20 .

161 Mitochondrial metabolism offers ECs a flexibility to utilize substrates for energy production, 162 which may become essential for ECs in disease conditions. For instance, hyperproliferative TECs utilize 163 OXPHOS for both biomass synthesis and energy production in glucose-deprived conditions. Indeed, in 164 tumors, glucose levels can be as low as $0.2 \mathrm{mM}$ (in contrast to $5.5 \mathrm{mM}$ in the blood) ${ }^{51}$. Accordingly, 165 proliferating TECs are more sensitive to inhibition of OXPHOS than quiescent ECs ${ }^{52-54}$. Moreover, ab166 errant mitochondrial metabolism is a hallmark of hyperproliferative PAH-ECs, which rely more on gly167 colysis for energy production, forfeiting their metabolic flexibility to some extent. PAH-ECs feature 168 reduced mitochondrial ATP production and volume, but increased ROS formation ${ }^{55,56}$.

\section{Fatty Acid Metabolism}

170 Lipids are used by ECs as (i) structural membrane components in the form of phospholipids ${ }^{57}$, (ii) as 171 signaling molecules mediating cellular responses such as growth, stress and apoptosis ${ }^{58,59}$, and (iii) to 172 sustain the TCA cycle (in conjunction with other anaplerotic substrates) ${ }^{37}$. ECs can take up fatty acids 173 (FAs) directly from the circulation, either passively or by designated transporters (CD36; FATP family), 174 and thereby regulate the lipid content in the blood, an important risk factor of atherosclerosis ${ }^{60}$. De- 
175 spite having access to circulating lipids, ECs can also synthesize FAs endogenously by converting ace176 tyl-CoA to malonyl-CoA, which is then elongated to FAs (Fig. 2) ${ }^{61}$.

177 During angiogenesis, the availability of FAs for ECs is essential ${ }^{62}$, and growth factor VEGF-B 178 has been shown to induce FA uptake by upregulation of FATP3 and FATP4 expression ${ }^{63}$. In addition, 179 the synthesis of FAs is upregulated in proliferating ECs ${ }^{19}$ and PAH-ECs ${ }^{64}$, making manipulation of this 180 pathway a potential target to treat pathological angiogenesis. Indeed, blocking FA synthesis by inhibi181 tion of acetyl-CoA carboxylase ACC (by soraphen A or genetic inactivation), the enzyme catalyzing the 182 reaction from acetyl-CoA to malonyl-CoA, decreases EC migration ${ }^{65}$. Mechanistically, the reduced 183 malonyl-CoA levels impede FA- and subsequently phospholipid synthesis ${ }^{66}$, leading to a shift in the 184 membrane phospholipid composition that affects membrane fluidity and filopodia formation (Fig. 185 2,3). Inhibiting fatty acid synthase (FASN), which is crucial for FA elongation from malonyl-CoA, de186 creases EC migration and/or proliferation ${ }^{61,67}$. Unexpectedly, since the angiogenesis defect cannot be 187 rescued by palmitate supplementation ${ }^{61}$, this defect is not a consequence of FA depletion, as ob188 served in cancer cells ${ }^{68}$. Instead, blockade of fatty acid synthesis leads to changes in posttranslational protein modifications, i.e. reduced eNOS palmitoylation or increased mTOR malonylation, which reduces their activity and impairs vascular sprouting (Fig. 2,3) ${ }^{61,67}$.

FAs intended for different purposes (storage, elongation or oxidation) are shuttled between 192 organelles by fatty acid transporters. Interestingly, the fatty acid transporter FABP4 is upregulated in 193 angiogenesis, while its inhibition in ECs perturbs FA metabolism (by lipolysis of stored FAs, which in194 creases the intracellular FA content and FAO) and induces oxidative stress, leading to reduced EC mi195 gration and proliferation ${ }^{69,70}$.

For catabolic use, FAs are imported into mitochondria by carnitine palmitoyl transferase 1a 197 (CPT1a), where they are oxidized and enter the TCA cycle in the form of acetyl-CoA (Fig. 2). Whereas 
198

199

200

201

202

203

204

205

206

207

208

209

210

211

212

213

214

215

216

217

218

219

220

many other cell types use FAO for energy production ${ }^{71-73}$, FAO in proliferating blood vascular and lymphatic ECs is essential for sustaining the TCA cycle (in conjunction with other anaplerotic substrates), which results in the synthesis of aspartate, a precursor of nucleotides needed for DNA replication during EC proliferation (Fig. 3) - a dependency rarely observed in other cell types ${ }^{37,74}$. Importantly, the TCA cycle in ECs is not replenished by a net contribution of FA-derived carbons in the traditional meaning of anaplerosis ${ }^{75}$. Although ${ }^{13} \mathrm{C}$-labelled acetyl-CoA derived from the FA palmitate enters the TCA cycle and labels oxaloacetate, there is no net formation of oxaloacetate from acetylCoA, derived from FAs. Rather, in conditions of sufficient availability of anaplerotic substrates (glutamine, glucose), FAO provides acetyl-CoA, which in conjunction with an anaplerotic substrate helps to sustain the TCA cycle, necessary for dNTP synthesis for proliferation. Blocking FAO by inhibiting CPT1a (by etomoxir) in angiogenic ECs reduces EC proliferation but not migration ${ }^{37}$. Supplementation with acetate, which can be converted to acetyl-CoA, or with nucleosides rescues the FAO defect ${ }^{37}$. In contrast to angiogenic ECs, quiescent ECs utilize FAO for NADPH regeneration via the TCA cycle (see Redox metabolism, Fig. 3) ${ }^{19}$

\section{FatTy ACID AND Ketone Body Metabolism in Lymphatic ECS}

Besides its importance in blood vascular ECs, CPT1a regulates lymphatic EC (LEC) development, not only by sustaining the TCA cycle and promoting nucleotide synthesis via the above described nonanaplerotic mechanism, but also by regulating venous-to-lymphatic EC differentiation via an epigenetic mechanism (Fig. 4) ${ }^{74,76}$. During embryonic development, LECs differentiate from venous ECs in a process driven by the transcription factor PROX1, which upregulates the expression of a lymphangiogenesis program, e.g. VEGF receptor 3 (VEGFR3) and others ${ }^{77}$. PROX1 binds to the CPT1a promotor and enhances its expression, leading to increased acetyl-CoA levels. Acetyl-CoA is then used for histone acetylation of PROX1-binding sites via direct interaction of PROX1 with the histone acetyltrans- 
221 ferase p300. As a result, PROX1 makes its target genes more accessible for itself, thereby potentiating 222 its own transcriptional activity ${ }^{74}$.

223 The fact that the lymphangiogenesis defect caused by FAO inhibition can be rescued by ace224 tate supplementation highlights the importance of acetyl-CoA for LECs. Primed by these results, a re225 cent study showed that acetyl-CoA derived from ketone body oxidation is crucial for lymphangiogen226 esis ${ }^{6}$. Ketone bodies are energy-rich metabolites secreted by the liver, which are oxidized in mito227 chondria of extra-hepatic tissues into two molecules of acetyl-CoA, which can then enter the TCA cy228 cle (Fig. 2) ${ }^{78}$. LEC-specific loss of 3-oxoacid-CoA-transferase-1 (OXCT1), a key enzyme of ketone body 229 oxidation $^{78}$, reduces LEC proliferation, migration and vessel sprouting in vitro and impairs lymphangi230 ogenesis in development and disease in Prox ${ }^{\Delta 0 x C T 1}$ mice, lacking Oxct1 in LECs ${ }^{76}$. Mechanistically, 231 OXCT1 silencing lowers acetyl-CoA levels, TCA cycle metabolite pools, and aspartate and dNTP levels, 232 required for LEC proliferation (Fig. 4). Ketone body supplementation to LECs induces opposite effects. 233 Notably, elevation of lymph ketone body levels in wild type (but no longer in Prox $1^{\Delta 0 \mathrm{xCT} 1}$ ) mice by a 234 high-fat, low-carbohydrate ketogenic diet or by administration of the ketone body $\beta$-hydroxybutyrate $235(\beta-\mathrm{OHB})$ increases lymphangiogenesis after corneal injury and myocardial infarction ${ }^{78}$. Intriguingly, in 236 a mouse model of microsurgical ablation of lymphatic vessels in the tail, which recapitulates features 237 of acquired lymphedema in humans, the ketogenic diet improved lymphatic vessel function and 238 growth, reduced infiltration of anti-lymphangiogenic immune cells, and decreased edema ${ }^{78}$, suggest239 ing a novel metabolite dietary therapeutic opportunity (see also Concluding Remarks) ${ }^{76}$.

\section{Glutamine AND Asparagine Metabolism}

241 Of the set of amino acids analyzed, cultured ECs consume glutamine (the most abundant non242 essential amino acid in the circulation) to the largest extent and use it, as many other cell types, not 243 only for protein and nucleotide synthesis but also as a carbon source to replenish the TCA cycle via 
244 glutaminase 1 (GLS1) (Fig. 2, 5) ${ }^{39,79}$. As such, GLS1 supports nucleotide synthesis and - to a certain 245 extent - citrate production and lipid biosynthesis via reductive carboxylation, though the latter con246 textual finding requires confirmation ${ }^{38,39}$. Glutaminase 2 , on the other hand, provides glutamine car247 bons and nitrogen for glutathione synthesis used for redox homeostasis in non-EC types, but its role 248 has not been studied in ECs yet (see Redox Metabolism). Accordingly, in in vitro and in vivo models, 249 glutamine catabolism blockade (by metabolic, genetic or pharmacological means) in ECs impairs pro250 tein and nucleotide synthesis, and renders ECs susceptible to oxidative stress, which leads to prolifer251 ation arrest and/or affects EC motility ${ }^{38,39}$.

252 Glutamine is essential for the synthesis of other amino acids and consequently proteins, and is 253 a nitrogen donor for the de novo synthesis of asparagine in ECs. Notably, supplementation of aspara254 gine plus $\alpha$-ketoglutarate rescued the phenotypes induced by glutamine starvation ${ }^{38,39}$. In contrast, 255 single agent supplementation with anti-oxidants or TCA cycle replenishment sufficed to rescue the 256 glutamine depletion-induced phenotype in cancer cells but not in ECs, suggesting a strong reliance of 257 ECs on glutamine ${ }^{39}$. This can be even more apparent in disease conditions, as for example ECs infect258 ed by Kaposi 's sarcoma-associated herpes virus depend on glutamine breakdown for survival ${ }^{80}$. As259 paragine is believed to serve as a signaling metabolite (rheostat) that senses the availability of TCA 260 intermediates for non-essential amino acid synthesis and coordinates a homeostatic response (Fig. 5) $261{ }^{81}$. Even though asparagine can be taken up by ECs, synthesis of this particular amino acid seems to be 262 critical, as inhibition of asparagine synthetase (ASNS, the enzyme producing asparagine from gluta263 mine and aspartate) impairs EC proliferation, even in conditions where asparagine is not limiting ${ }^{39}$.

264 Intriguingly, despite the high levels of glutamine in the circulation, ECs express glutamine syn265 thetase (GS), allowing them to synthesize glutamine from glutamate. However, even in glutamine266 deprived conditions (which upregulates GS expression), ECs still only synthesize negligible amounts of 267 glutamine ${ }^{82}$. Nonetheless, GS is of particular importance during vessel growth as EC loss of GS impairs 
268 EC migration, but not proliferation (Fig. 5) ${ }^{82}$. The underlying mechanism relates, at least in part, to 269 perturbed actin remodeling, caused by decreased localization in the plasma membrane of RhoJ, a Rho 270 GTPase enriched in ECs and an assembly regulator of cytoskeleton proteins ${ }^{83}$. RhoJ requires pal271 mitoylation for plasma membrane localization and activity, which is lost upon GS blockade. Molecular 272 characterization and structural modeling identified GS as a previously unrecognized palmitoyl trans273 ferase ${ }^{82}$. While RhoJ was one identified target of the palmitoyl transferase activity of GS, it is likely 274 that other targets are also affected.

\section{SERINe Metabolism}

276 ECs can take up serine from the extracellular milieu or synthesize it de novo from the glycolytic inter277 mediate 3-phosphoglycerate (3PG) (Fig. 2, 5) and utilize it for the synthesis of heme, glutathione and 278 nucleotides (dATP, dGTP, dTTP) ${ }^{84}$. The de novo synthesis pathway starts with a rate-controlling step 279 controlled by phosphoglycerate dehydrogenase (PHGDH) ${ }^{85}$. Loss of endothelial PHGDH impairs EC 280 proliferation and survival, causing severe vascular defects and neonatal lethality upon Phgdh gene 281 inactivation in ECs ${ }^{48}$. The underlying mechanism of the PHGDH inhibition defect is a depletion of the 282 cellular heme pool, which impedes the activity of heme-containing OXPHOS complexes III and IV, and 283 thus results in electron leakage and defective mitochondrial respiration (Fig. 5) ${ }^{48}$. Escaped electrons 284 react with oxygen, which - together with decreased glutathione levels - causes ROS accumulation 285 and oxidative stress, and induces EC death ${ }^{48}$. Moreover, PHGDH defective ECs display not only the 286 expected decrease in dATP, dGTP and dTTP levels, but also in dCTP levels (not documented in any 287 other cell type to date). This is due to an impaired activity of dihydroorotate dehydrogenase (DHODH) 288 - a mitochondrial enzyme crucial for dCTP synthesis, the activity of which is coupled to and depend289 ent on functional electron transport in OXPHOS. Treatment of ECs with a heme synthesis blocker 290 phenocopied the PHGDH loss, while supplementation with hemin rescued the proliferation defect in 
291 PHGDH defective ECs, thus illustrating the importance of the role of PHGDH-dependent serine syn292 thesis in heme production ${ }^{48}$. In contrast to other cell types ${ }^{48,86,87}$, ECs rely on de novo serine biosyn293 thesis in a peculiar manner, as PHGDH knockdown in ECs reduces cellular serine levels and induces 294 apoptosis, which cannot be rescued by supplementation with the amino acids glycine and serine, 295 even though they can take up serine from the extracellular medium ${ }^{48}$. In tumors, the flux of glycolytic 296 intermediates to serine biosynthesis is enhanced to sustain nucleotide synthesis for the hyperprolif297 erative TEC phenotype ${ }^{22,48}$. Consistently, TECs express elevated levels of PHGDH ${ }^{48}$, representing a 298 therapeutic vulnerability to be explored for new anti-angiogenic approaches.

\section{Redox Metabolism}

300 A balanced redox state is crucial for ECs (for reviews, see ${ }^{88,89}$ ). At physiological concentrations, ROS 301 serve as key mediators in cellular signaling regulating among others EC growth, survival and angio302 genesis ${ }^{90}$. Highly elevated levels of ROS, however, give rise to oxidative stress, reduce bioavailability 303 of the key vascular tone regulator nitric oxide (NO) and lead to EC activation and dysfunction, as has 304 for example been observed in ECs in atherosclerosis ${ }^{91}$.

305 Healthy quiescent ECs lining perfused vessels are exposed to a high oxygen environment, 306 which necessitates protective mechanisms against oxidative damage (Fig. 1). For this reason, upon 307 entering a quiescent state, ECs adapt their metabolism by increasing FAO (Fig. 3) ${ }^{19}$. A similar increase 308 in FAO was documented upon overexpression of FOXO1, which induces EC quiescence ${ }^{20}$. Interesting309 Iy, the anti-mitogenic signal Notch, which inhibits nucleotide synthesis, seems to be a major regulator 310 of controlling the switch between the use of FAO for promoting nucleotide synthesis in proliferating 311 ECs (low Notch) versus the use of FAO for redox homeostasis in quiescent ECs (high Notch) ${ }^{19}$. Since 312 proliferating ECs utilize FAO to support the TCA cycle for nucleotide synthesis (see above), it is surpris313 ing that quiescent ECs would have higher, not lower FAO levels, since quiescent ECs synthesize fewer 
314 nucleotides as they proliferate less. Intriguingly, the function of FAO in quiescent ECs is markedly dif315 ferent. Instead of being used for energy production or TCA cycle support for biomass synthesis, FAO is 316 used to sustain redox homeostasis in quiescent ECs ${ }^{19}$. FA-derived acetyl-CoA is used to sustain the 317 TCA cycle, which generates isocitrate and malate, i.e. substrates of NADPH-producing malic enzyme 318 and isocitrate dehydrogenase (IDH2, Fig. 2), the deficiency of which causes oxidative stress in ECs and 319 vascular inflammation ${ }^{92}$. Indeed, NADPH is used to regenerate reduced glutathione (GSH) from its 320 oxidized form (GSSG) by glutathione reductase (see below) ${ }^{93}$. At the same time, when ECs switch 321 from proliferation to quiescence, they upregulate the expression of other NADPH-generating pathways and vasculoprotective proteins that consume NADPH (e.g. G6PDH, glutaredoxin-2 (GLRX2), glu323 tathione peroxidase 3 (GPx3), peroxiredoxin-1 (PRDX1) and eNOS, Fig. 3) ${ }^{19}$. Hence, to meet the in324 creased demands of NADPH by vasculoprotective genes, quiescent ECs reprogram their metabolism 325 to produce more NADPH via several routes, including increased FAO. The increased demands of anti326 oxidant protection thus seem to relate to the fact that quiescent ECs are exposed to high oxygen lev327 els in the blood stream and hence are in need of mechanisms to protect them from oxidative stress.

This may explain why EC loss of CPT1a aggravates inflammatory bowel disease due to elevated ROS levels ${ }^{19}$. Indeed, EC loss of CPT1a or its pharmacological inhibition in vivo induces vascular leakage and EC hyperpermeability (by affecting intracellular $\mathrm{Ca}^{2+}$ homeostasis) and leads to EC dysfunction ${ }^{94,95}$. Of interest, treatment of mice, lacking CPT1a in ECs, with acetate (a source of acetyl-CoA) 332 protects them against ROS production, vascular inflammation and EC dysfunction ${ }^{19}$, raising the ques333 tion whether such type of metabolite delivery might be useful for the treatment of vascular complica334 tions in diabetes, atherosclerosis, etc.

335 Several other metabolic pathways are involved in the protection against oxidative damage in 336 ECs: (i) by relying mostly on glycolytic metabolism, ECs limit the levels of ROS produced by OXPHOS; 337 (ii) both glutamine and glycine are required for glutathione synthesis and, thus, deprivation of gluta- 
338 mine or serine (which is converted to glycine via serine hydroxymethyltransferase) are associated 339 with increased ROS levels (see Serine Metabolism) ${ }^{39,48}$; (iii) serine, as one-carbon donor for the folate 340 cycle, contributes to the generation of NADPH; (iv) UbiA prenyltransferase domain-containing protein 3411 (UBIAD1), which synthesizes the eNOS cofactor Coenzyme Q10, protects ECs from oxidative stress 342 and ROS-mediated lipid peroxidation ${ }^{96}$.

\section{CONCLUDING REMARKS \& THERAPEUTIC PERSPECTIVES}

344 Studies during the past decade highlighted the importance of EC metabolism in vessel sprouting in 345 vitro and in vivo, and illustrated that manipulating EC metabolism can overrule instructions by 346 (lymph)angiogenic signals. While not only providing new fundamental insights in EC biology, these 347 findings also raise the question whether they can serve for the development of novel therapeutic 348 strategies to promote or inhibit growth of blood vascular or lymphatic vessels. We will highlight two 349 complementary examples. First, as an example of an approach to inhibit a metabolic target in ECs, we 350 discuss the potential of blockade of the glycolytic activator PFKFB3 in glycolysis-addicted TECs (Fig. 351 6A). While a justified concern is that not only TECs but also other healthy ECs and non-EC types may 352 require glycolysis, TECs rely on glycolysis more than other cell types, which can switch to other meta353 bolic pathways more readily upon glycolysis blockade; precisely therefore, they are more sensitive to 354 even slight reductions in glycolysis levels ${ }^{22}$. The therapeutic goal is not to eliminate glycolysis (which 355 would cause toxic side-effects in other cell types) but rather to normalize the hyperglycolysis back to 356 glycolysis levels in quiescent ECs, so that TECs do no longer grow ${ }^{97}$. In fact, lowering TEC glycolysis by 357 15-25\% sufficed to normalize tumor vessel abnormalities, thereby reducing metastasis and improving 358 chemotherapy ${ }^{22}$, without inducing systemic toxicity (because such a low level of glycolysis inhibition 359 is readily compensated by cell types that are not addicted to glycolysis). In contrast, lowering TEC gly360 colysis by a maximally tolerable anti-glycolytic activator dose induced opposite effects due to tumor 
361 vessel disintegration ${ }^{27}$. Clinical development of an inhibitor of a glycolytic activator will be required 362 to prove this hypothesis. Silencing or blocking other EC metabolic targets (hexokinase $2^{21} ;$ PKM2 ${ }^{29}$; $363 \mathrm{GP}{ }^{30}$; $\mathrm{PHGDH}^{48}$; $\mathrm{CPT} 1 \mathrm{a}^{37}$; ACC1 $^{65}$; FAPB4 ${ }^{69,70}$; $\mathrm{GS}^{82}$; GLS1 ${ }^{38,39}$; G6PDH ${ }^{30,32}$; FASN ${ }^{61,67}$; OXCT1 ${ }^{76}$; 364 UQCRQ ${ }^{47} ; \mathrm{FADH}^{49}$ ) has been shown to inhibit vessel sprouting in vitro and/or in vivo. These targets 365 might deserve future attention for possible AAT development, whereby it would be preferable to de366 velop strategies to target such drugs to the ECs in neovessels in diseased tissues, while sparing normal 367 quiescent ECs in healthy tissues. Indeed, studies showing that, for instance, the inhibition of PKM2 368 impairs the vascular barrier integrity of quiescent ECs and that CPT1a inhibition leads to oxidative 369 stress and dysfunction of quiescent ECs urge for caution when developing EC metabolism-targeted 370 therapeutics ${ }^{19,98}$.

Second, a ketogenic diet showed a promising improvement of lymphedema in a preclinical 372 mouse model, a strategy rationally deduced from obtaining fundamental insights on how lipids and 373 ketone bodies affect LEC biology ${ }^{74,76}$. This has resulted in the initiation of a phase II clinical trial to 374 test the clinical effect of dietary ketone bodies (high fat/low carbohydrate ketogenic diet) in 375 lymphedema patients (Fig. 6B).

Despite recent advances, only few metabolic enzymes have been characterized in ECs to date 377 in sufficient detail. Further research is needed to uncover complex metabolic links and to identify 378 other pathways that can be targeted to control angiogenesis or EC dysfunction. Considering the func379 tional heterogeneity of ECs in different states (quiescent, stalk, tip cells), vascular beds (arterial, ve380 nous, capillary, lymphatic vessels) and tissues, multi-omics profiling of ECs including at the single cell 381 level could prove to be invaluable for future development of EC-targeted therapies. Advanced bioin382 formatics tools allowing easy dataset exploration, like EndoDB ${ }^{99}$, and in silico approaches like compu383 tational genome-scale metabolic modelling of ECs can further help facilitate the discovery of novel 384 unexplored targets and promise to yield exciting new perspective in the field. 


\section{ACKNOWLEDGEMENTS}

387 The authors apologize to any author whose work could not be included due to space limitations, and 388 thank all lab members, colleagues and collaborators for contributing to the discussed data. KF is sup389 ported by a Marie Sklodowska-Curie IF (H2020-MSCA-IF-2017, No. 799522). KR is supported by the 390 Research Foundation Flanders (FWO, grant no. 12V9318N). YL is supported by Sanming Project of 391 Medicine in Shenzhen (SZSM201612074), by BGI-Research, Danish Research Council for Independent 392 Research (DFF-1337-00128), Sapere Aude Young Research Talent Prize (DFF-1335-00763A) and Aar393 hus University Strategic Grant (AU-iCRISPR). The work of PC is supported by a Belgian Science Policy 394 grant (IUAP7/03), long-term structural Methusalem funding by the Flemish Government, grants from 395 the FWO (G.0834.13N and G.0532.10N), Foundation against Cancer (grant no. 2012-175), a European 396 Research Council (ERC) Advanced Research Grant (EU ERC269073) and an AXA Research grant. 397

398 AUTHOR CONTRIBUTIONS: All authors prepared, revised the manuscript and approved the final version; 399 KF prepared the figures.

400

401 CONFLICT OF INTEREST DECLARATION: The authors declare no competing financial interests. 402 403 AUTHOR INFORMATION: Correspondence should be addressed to peter.carmeliet@kuleuven.vib.be. 404 405 
406

407

408

409

410

411

412

413

414

415

416

417

418

419

420

421

422

423

424

425

426

427

428

429

430

431

432

433

434

435

436

437

438

439

440

441

442

443

444

\section{REFERENCES}

1 Potente, M., Gerhardt, H. \& Carmeliet, P. Basic and therapeutic aspects of angiogenesis. Cell 146, 873-887, doi:10.1016/j.cell.2011.08.039 (2011).

2 Carmeliet, P. \& Jain, R. K. Molecular mechanisms and clinical applications of angiogenesis. Nature 473, 298-307, doi:10.1038/nature10144 (2011).

3 Eelen, G. et al. Endothelial Cell Metabolism. Physiological reviews 98, 3-58, doi:10.1152/physrev.00001.2017 (2018).

4 Jakobsson, L. et al. Endothelial cells dynamically compete for the tip cell position during angiogenic sprouting. Nature cell biology 12, 943-953, doi:10.1038/ncb2103 (2010).

5 Tabit, C. E., Chung, W. B., Hamburg, N. M. \& Vita, J. A. Endothelial dysfunction in diabetes mellitus: molecular mechanisms and clinical implications. Reviews in endocrine \& metabolic disorders 11, 61-74, doi:10.1007/s11154-010-9134-4 (2010).

6 Gimbrone, M. A., Jr. \& Garcia-Cardena, G. Endothelial Cell Dysfunction and the Pathobiology of Atherosclerosis. Circ Res 118, 620-636, doi:10.1161/circresaha.115.306301 (2016).

7 Vasa, M. et al. Number and migratory activity of circulating endothelial progenitor cells inversely correlate with risk factors for coronary artery disease. Circ Res 89, E1-7 (2001).

8 Matsuzawa, Y. \& Lerman, A. Endothelial dysfunction and coronary artery disease: assessment, prognosis, and treatment. Coronary artery disease 25, 713-724, doi:10.1097/mca.0000000000000178 (2014).

9 Gutierrez, E. et al. Endothelial dysfunction over the course of coronary artery disease. Eur Heart J 34, 3175-3181, doi:10.1093/eurheartj/eht351 (2013).

10 Chrissobolis, S., Miller, A. A., Drummond, G. R., Kemp-Harper, B. K. \& Sobey, C. G. Oxidative stress and endothelial dysfunction in cerebrovascular disease. Frontiers in bioscience (Landmark edition) 16, 1733-1745 (2011).

11 Malyszko, J. Mechanism of endothelial dysfunction in chronic kidney disease. Clinica chimica acta; international journal of clinical chemistry 411, 1412-1420, doi:10.1016/j.cca.2010.06.019 (2010).

12 Campochiaro, P. A. Ocular neovascularization. Journal of molecular medicine (Berlin, Germany) 91, 311-321, doi:10.1007/s00109-013-0993-5 (2013).

13 Folkman, J. Role of angiogenesis in tumor growth and metastasis. Seminars in oncology 29, 15-18, doi:10.1053/sonc.2002.37263 (2002).

14 Voelkel, N. F. \& Gomez-Arroyo, J. The role of vascular endothelial growth factor in pulmonary arterial hypertension. The angiogenesis paradox. American journal of respiratory cell and molecular biology 51, 474-484, doi:10.1165/rcmb.2014-0045TR (2014).

15 Ebos, J. M. \& Kerbel, R. S. Antiangiogenic therapy: impact on invasion, disease progression, and metastasis. Nature reviews. Clinical oncology 8, 210-221, doi:10.1038/nrclinonc.2011.21 (2011).

16 Aragones, J., Fraisl, P., Baes, M. \& Carmeliet, P. Oxygen sensors at the crossroad of metabolism. Cell metabolism 9, 11-22, doi:10.1016/j.cmet.2008.10.001 (2009). 
445

446

447

448

449

450

451

452

453

454

455

456

457

458

459

460

461

462

463

464

465

466

467

468

469

470

471

472

473

474

475

476

477

478

479

480

481

482

483

484

17 De Bock, K. et al. Role of PFKFB3-driven glycolysis in vessel sprouting. Cell 154, 651663, doi:10.1016/j.cell.2013.06.037 (2013).

18 Mertens, S., Noll, T., Spahr, R., Krutzfeldt, A. \& Piper, H. M. Energetic response of coronary endothelial cells to hypoxia. Am J Physiol 258, H689-694, doi:10.1152/ajpheart.1990.258.3.H689 (1990).

19 Kalucka, J. et al. Quiescent Endothelial Cells Upregulate Fatty Acid beta-Oxidation for Vasculoprotection via Redox Homeostasis. Cell metabolism 28, 881-894.e813, doi:10.1016/j.cmet.2018.07.016 (2018).

20 Wilhelm, K. et al. FOXO1 couples metabolic activity and growth state in the vascular endothelium. Nature 529, 216-220, doi:10.1038/nature16498 (2016).

$21 \mathrm{Yu}$, P. et al. FGF-dependent metabolic control of vascular development. Nature 545, 224-228, doi:10.1038/nature22322 (2017).

22 Cantelmo, A. R. et al. Inhibition of the Glycolytic Activator PFKFB3 in Endothelium Induces Tumor Vessel Normalization, Impairs Metastasis, and Improves Chemotherapy. Cancer cell 30, 968-985, doi:10.1016/j.ccell.2016.10.006 (2016).

23 Thenappan, T., Ormiston, M. L., Ryan, J. J. \& Archer, S. L. Pulmonary arterial hypertension: pathogenesis and clinical management. BMJ (Clinical research ed.) 360, j5492, doi:10.1136/bmj.j5492 (2018).

24 Cruys, B. et al. Glycolytic regulation of cell rearrangement in angiogenesis. Nature communications 7, 12240, doi:10.1038/ncomms12240 (2016).

$25 \mathrm{Xu}, \mathrm{Y}$. et al. Endothelial PFKFB3 plays a critical role in angiogenesis. Arteriosclerosis, thrombosis, and vascular biology 34, 1231-1239, doi:10.1161/atvbaha.113.303041 (2014).

26 Schoors, S. et al. Partial and transient reduction of glycolysis by PFKFB3 blockade reduces pathological angiogenesis. Cell metabolism 19, 37-48, doi:10.1016/j.cmet.2013.11.008 (2014).

27 Conradi, L. C. et al. Tumor vessel disintegration by maximum tolerable PFKFB3 blockade. Angiogenesis, doi:10.1007/s10456-017-9573-6 (2017).

28 Liu, Z. et al. Endothelial adenosine A2a receptor-mediated glycolysis is essential for pathological retinal angiogenesis. Nat Commun 8, 584, doi:10.1038/s41467-017-00551-2 (2017).

29 Stone, $\mathrm{O}$. A. et al. Loss of pyruvate kinase M2 limits growth and triggers innate immune signaling in endothelial cells. Nature communications 9, 4077, doi:10.1038/s41467-018-06406-8 (2018).

30 Vizan, P. et al. Characterization of the metabolic changes underlying growth factor angiogenic activation: identification of new potential therapeutic targets. Carcinogenesis 30, 946-952, doi:10.1093/carcin/bgp083 (2009).

31 Forstermann, U. \& Sessa, W. C. Nitric oxide synthases: regulation and function. Eur Heart J 33, 829-837, 837a-837d, doi:10.1093/eurheartj/ehr304 (2012).

32 Leopold, J. A. et al. Glucose-6-phosphate dehydrogenase modulates vascular endothelial growth factor-mediated angiogenesis. The Journal of biological chemistry 278, 3210032106, doi:10.1074/jbc.M301293200 (2003). 
485

486

487

488

489

490

491

492

493

494

495

496

497

498

499

500

501

502

503

504

505

506

507

508

509

510

511

512

513

514

515

516

517

518

519

520

521

522

523

524

33 Fessel, J. P. et al. Metabolomic analysis of bone morphogenetic protein receptor type 2 mutations in human pulmonary endothelium reveals widespread metabolic reprogramming. Pulm Circ 2, 201-213, doi:10.4103/2045-8932.97606 (2012).

34 Chandler, K. B., Leon, D. R., Meyer, R. D., Rahimi, N. \& Costello, C. E. Site-Specific NGlycosylation of Endothelial Cell Receptor Tyrosine Kinase VEGFR-2. Journal of proteome research 16, 677-688, doi:10.1021/acs.jproteome.6b00738 (2017).

35 Zibrova, D. et al. GFAT1 phosphorylation by AMPK promotes VEGF-induced angiogenesis. The Biochemical journal 474, 983-1001, doi:10.1042/bcj20160980 (2017).

36 Luo, B., Soesanto, Y. \& McClain, D. A. Protein modification by O-linked GIcNAc reduces angiogenesis by inhibiting Akt activity in endothelial cells. Arteriosclerosis, thrombosis, and vascular biology 28, 651-657, doi:10.1161/atvbaha.107.159533 (2008).

37 Schoors, S. et al. Fatty acid carbon is essential for dNTP synthesis in endothelial cells. Nature 520, 192-197, doi:10.1038/nature14362 (2015).

38 Kim, B., Li, J., Jang, C. \& Arany, Z. Glutamine fuels proliferation but not migration of endothelial cells. EMBO J 36, 2321-2333, doi:10.15252/embj.201796436 (2017).

39 Huang, $\mathrm{H}$. et al. Role of glutamine and interlinked asparagine metabolism in vessel formation. $E M B O$ J 36, 2334-2352, doi:10.15252/embj.201695518 (2017).

40 Blouin, A., Bolender, R. P. \& Weibel, E. R. Distribution of organelles and membranes between hepatocytes and nonhepatocytes in the rat liver parenchyma. A stereological study. The Journal of cell biology 72, 441-455 (1977).

41 Oldendorf, W. H., Cornford, M. E. \& Brown, W. J. The large apparent work capability of the blood-brain barrier: a study of the mitochondrial content of capillary endothelial cells in brain and other tissues of the rat. Annals of neurology 1, 409-417, doi:10.1002/ana.410010502 (1977).

42 Groschner, L. N., Waldeck-Weiermair, M., Malli, R. \& Graier, W. F. Endothelial mitochondria--less respiration, more integration. Pflugers Arch 464, 63-76, doi:10.1007/s00424-0121085-z (2012).

43 Kluge, M. A., Fetterman, J. L. \& Vita, J. A. Mitochondria and endothelial function. Circ Res 112, 1171-1188, doi:10.1161/CIRCRESAHA.111.300233 (2013).

44 Kadlec, A. O., Beyer, A. M., Ait-Aissa, K. \& Gutterman, D. D. Mitochondrial signaling in the vascular endothelium: beyond reactive oxygen species. Basic Res Cardiol 111, 26, doi:10.1007/s00395-016-0546-5 (2016).

45 Marcu, R., Zheng, Y. \& Hawkins, B. J. Mitochondria and Angiogenesis. Adv Exp Med Biol 982, 371-406, doi:10.1007/978-3-319-55330-6_21 (2017).

46 Koziel, A., Woyda-Ploszczyca, A., Kicinska, A. \& Jarmuszkiewicz, W. The influence of high glucose on the aerobic metabolism of endothelial EA.hy926 cells. Pflugers Arch 464, 657-669, doi:10.1007/s00424-012-1156-1 (2012).

47 Diebold, L. P. et al. Mitochondrial complex III is necessary for endothelial cell proliferation during angiogenesis. Nature Metabolism 1, 158-171 (2019).

48 Vandekeere, S. et al. Serine Synthesis via PHGDH Is Essential for Heme Production in Endothelial Cells. Cell metabolism 28, 573-587 e513, doi:10.1016/j.cmet.2018.06.009 (2018). 
532

533

534

535

536

537

538

539

540

541

542

543

544

545

546

547

548

549

550

551

552

553

554

555

556

557

558

559

560

561

562

563

564

49 Petit, M., Koziel, R., Etemad, S., Pircher, H. \& Jansen-Durr, P. Depletion of oxaloacetate decarboxylase FAHD1 inhibits mitochondrial electron transport and induces cellular senescence in human endothelial cells. Exp Gerontol 92, 7-12, doi:10.1016/j.exger.2017.03.004 (2017).

50 Andrade, J. \& Potente, M. Endothelial metabolism-more complex (III) than previously thought. Nature Metabolism 1, 14-15, doi:10.1038/s42255-018-0019-2 (2019).

51 Hirayama, A. et al. Quantitative metabolome profiling of colon and stomach cancer microenvironment by capillary electrophoresis time-of-flight mass spectrometry. Cancer research 69, 4918-4925, doi:10.1158/0008-5472.Can-08-4806 (2009).

52 Coutelle, O. et al. Embelin inhibits endothelial mitochondrial respiration and impairs neoangiogenesis during tumor growth and wound healing. EMBO molecular medicine 6, 624-639, doi:10.1002/emmm.201303016 (2014).

53 Don, A. S. et al. A peptide trivalent arsenical inhibits tumor angiogenesis by perturbing mitochondrial function in angiogenic endothelial cells. Cancer cell 3, 497-509 (2003).

54 Blecha, J. et al. Antioxidant defense in quiescent cells determines selectivity of electron transport chain inhibition-induced cell death. Free radical biology \& medicine 112, 253-266, doi:10.1016/j.freeradbiomed.2017.07.033 (2017).

$55 \mathrm{Xu}, \mathrm{W}$. et al. Alterations of cellular bioenergetics in pulmonary artery endothelial cells. Proceedings of the National Academy of Sciences of the United States of America 104, 1342-1347, doi:10.1073/pnas.0605080104 (2007).

56 Diebold, I. et al. BMPR2 preserves mitochondrial function and DNA during reoxygenation to promote endothelial cell survival and reverse pulmonary hypertension. Cell metabolism 21, 596-608, doi:10.1016/j.cmet.2015.03.010 (2015).

57 Spector, A. A. \& Yorek, M. A. Membrane lipid composition and cellular function. Journal of lipid research 26, 1015-1035 (1985).

58 O'Donnell, V. B. Free Radicals and Lipid Signaling in Endothelial Cells. Antioxidants \& redox signaling 5, 195-203, doi:10.1089/152308603764816550 (2003).

59 Wymann, M. P. \& Schneiter, R. Lipid signalling in disease. Nat Rev Mol Cell Biol 9, 162176, doi:10.1038/nrm2335 (2008).

60 Ghosh, A., Gao, L., Thakur, A., Siu, P. M. \& Lai, C. W. K. Role of free fatty acids in endothelial dysfunction. Journal of biomedical science 24, 50, doi:10.1186/s12929-017-0357-5 (2017).

61 Bruning, U. et al. Impairment of Angiogenesis by Fatty Acid Synthase Inhibition Involves mTOR Malonylation. Cell metabolism 28, 866-880.e815, doi:10.1016/j.cmet.2018.07.019 (2018).

62 Vanetti, C., Bifari, F., Vicentini, L. M. \& Cattaneo, M. G. Fatty acids rather than hormones restore in vitro angiogenesis in human male and female endothelial cells cultured in charcoal-stripped serum. PLoS One 12, e0189528, doi:10.1371/journal.pone.0189528 (2017).

63 Hagberg, C. E. et al. Vascular endothelial growth factor B controls endothelial fatty acid uptake. Nature 464, 917-921, doi:10.1038/nature08945 (2010).

64 Singh, N., Singh, H., Jagavelu, K., Wahajuddin, M. \& Hanif, K. Fatty acid synthase modulates proliferation, metabolic functions and angiogenesis in hypoxic pulmonary artery endothelial cells. Eur J Pharmacol 815, 462-469, doi:10.1016/j.ejphar.2017.09.042 (2017). 
565

566

567

568

569

570

571

572

573

574

575

576

577

578

579

580

581

582

583

584

585

586

587

588

589

590

591

592

593

594

595

596

597

598

599

600

601

602

603

604

605

65 Glatzel, D. K. et al. Acetyl-CoA carboxylase 1 regulates endothelial cell migration by shifting the phospholipid composition. J Lipid Res 59, 298-311, doi:10.1194/jlr.M080101 (2018).

66 Swinnen, J. V. et al. Fatty acid synthase drives the synthesis of phospholipids partitioning into detergent-resistant membrane microdomains. Biochemical and biophysical research communications 302, 898-903 (2003).

67 Wei, X. et al. De novo lipogenesis maintains vascular homeostasis through endothelial nitric-oxide synthase (eNOS) palmitoylation. The Journal of biological chemistry 286, 2933-2945, doi:10.1074/jbc.M110.193037 (2011).

68 Ventura, R. et al. Inhibition of de novo Palmitate Synthesis by Fatty Acid Synthase Induces Apoptosis in Tumor Cells by Remodeling Cell Membranes, Inhibiting Signaling Pathways, and Reprogramming Gene Expression. EBioMedicine 2, 808-824, doi:10.1016/j.ebiom.2015.06.020 (2015).

69 Elmasri, H. et al. Endothelial cell-fatty acid binding protein 4 promotes angiogenesis: role of stem cell factor/c-kit pathway. Angiogenesis 15, 457-468, doi:10.1007/s10456-012-9274-0 (2012).

70 Elmasri, H. et al. Fatty acid binding protein 4 is a target of VEGF and a regulator of cell proliferation in endothelial cells. Faseb j 23, 3865-3873, doi:10.1096/fj.09-134882 (2009).

71 Ghesquiere, B., Wong, B. W., Kuchnio, A. \& Carmeliet, P. Metabolism of stromal and immune cells in health and disease. Nature 511, 167-176, doi:10.1038/nature13312 (2014).

72 Qu, Q., Zeng, F., Liu, X., Wang, Q. J. \& Deng, F. Fatty acid oxidation and carnitine palmitoyltransferase I: emerging therapeutic targets in cancer. Cell death \& disease 7, e2226, doi:10.1038/cddis.2016.132 (2016).

73 Stoll, E. A. et al. Neural Stem Cells in the Adult Subventricular Zone Oxidize Fatty Acids to Produce Energy and Support Neurogenic Activity. Stem cells (Dayton, Ohio) 33, 2306-2319, doi:10.1002/stem.2042 (2015).

74 Wong, B. W. et al. The role of fatty acid beta-oxidation in lymphangiogenesis. Nature, doi:10.1038/nature21028 (2016).

75 Schoors, S. et al. Corrigendum: Fatty acid carbon is essential for dNTP synthesis in endothelial cells. Nature 526, 144, doi:10.1038/nature14624 (2015).

76 García-Caballero, M. et al. Role and therapeutic potential of dietary ketone bodies for lymph vessel growth. Nature Metabolism (2019, in press).

77 Wong, B. W., Zecchin, A., Garcia-Caballero, M. \& Carmeliet, P. Emerging Concepts in Organ-Specific Lymphatic Vessels and Metabolic Regulation of Lymphatic Development. Dev Cell 45, 289-301, doi:10.1016/j.devcel.2018.03.021 (2018).

78 Puchalska, P. \& Crawford, P. A. Multi-dimensional Roles of Ketone Bodies in Fuel Metabolism, Signaling, and Therapeutics. Cell Metab 25, 262-284, doi:10.1016/j.cmet.2016.12.022 (2017).

79 DeBerardinis, R. J. \& Cheng, T. Q's next: the diverse functions of glutamine in metabolism, cell biology and cancer. Oncogene 29, 313-324, doi:10.1038/onc.2009.358 (2010).

80 Sanchez, E. L., Carroll, P. A., Thalhofer, A. B. \& Lagunoff, M. Latent KSHV Infected Endothelial Cells Are Glutamine Addicted and Require Glutaminolysis for Survival. PLoS pathogens 11, e1005052, doi:10.1371/journal.ppat.1005052 (2015). 
606

607

608 609

610

611

612

613

614

615

616

617

618

619

620

621

622

623

624

625

626

627

628

629

630

631

632

633

634

635

636

637

638

639

640

641

642

643

644

645

646

81 Zhang, J. et al. Asparagine plays a critical role in regulating cellular adaptation to glutamine depletion. Mol. Cell 56, 205-218, doi:10.1016/j.molcel.2014.08.018 (2014).

82 Eelen, G. et al. Role of glutamine synthetase in angiogenesis beyond glutamine synthesis. Nature 561, 63-69, doi:10.1038/s41586-018-0466-7 (2018).

83 Yuan, L. et al. RhoJ is an endothelial cell-restricted Rho GTPase that mediates vascular morphogenesis and is regulated by the transcription factor ERG. Blood 118, 1145-1153, doi:10.1182/blood-2010-10-315275 (2011).

84 Lane, A. N. \& Fan, T. W. Regulation of mammalian nucleotide metabolism and biosynthesis. Nucleic acids research 43, 2466-2485, doi:10.1093/nar/gkv047 (2015).

85 Amelio, I., Cutruzzola, F., Antonov, A., Agostini, M. \& Melino, G. Serine and glycine metabolism in cancer. Trends in biochemical sciences 39, 191-198, doi:10.1016/j.tibs.2014.02.004 (2014).

86 Zhang, T. et al. Disruption of De Novo Serine Synthesis in Muller Cells Induced Mitochondrial Dysfunction and Aggravated Oxidative Damage. Molecular neurobiology 55, 70257037, doi:10.1007/s12035-017-0840-8 (2018).

87 Possemato, R. et al. Functional genomics reveal that the serine synthesis pathway is essential in breast cancer. Nature 476, 346, doi:10.1038/nature10350 (2011).

88 Santoro, M. M. Fashioning blood vessels by ROS signalling and metabolism. Semin Cell Dev Biol 80, 35-42, doi:10.1016/j.semcdb.2017.08.002 (2018).

89 Schroder, K. Redox Control of Angiogenesis. Antioxidants \& redox signaling, doi:10.1089/ars.2017.7429 (2018).

90 Breton-Romero, R. \& Lamas, S. Hydrogen peroxide signaling in vascular endothelial cells. Redox biology 2, 529-534, doi:10.1016/j.redox.2014.02.005 (2014).

91 Incalza, M. A. et al. Oxidative stress and reactive oxygen species in endothelial dysfunction associated with cardiovascular and metabolic diseases. Vascular pharmacology 100, 1-19, doi:10.1016/j.vph.2017.05.005 (2018).

92 Choi, S. J. et al. Isocitrate dehydrogenase 2 deficiency induces endothelial inflammation via p66sh-mediated mitochondrial oxidative stress. Biochemical and biophysical research communications 503, 1805-1811, doi:10.1016/j.bbrc.2018.07.117 (2018).

93 Forman, H. J., Zhang, H. \& Rinna, A. Glutathione: overview of its protective roles, measurement, and biosynthesis. Mol Aspects Med 30, 1-12, doi:10.1016/j.mam.2008.08.006 (2009).

$94 \quad$ Patella, F. et al. Proteomics-based metabolic modeling reveals that fatty acid oxidation (FAO) controls endothelial cell (EC) permeability. Mol Cell Proteomics 14, 621-634, doi:10.1074/mcp.M114.045575 (2015).

95 Kalucka, J. et al. Quiescent Endothelial Cells Upregulate Fatty Acid beta-Oxidation for Vasculoprotection via Redox Homeostasis. Cell Metab, doi:10.1016/j.cmet.2018.07.016 (2018).

96 Mugoni, V. et al. Ubiad1 is an antioxidant enzyme that regulates eNOS activity by CoQ10 synthesis. Cell 152, 504-518, doi:10.1016/j.cell.2013.01.013 (2013).

97 Carmeliet, P. \& Jain, R. K. Principles and mechanisms of vessel normalization for cancer and other angiogenic diseases. Nature reviews. Drug discovery 10, 417-427, doi:10.1038/nrd3455 (2011). 
$647 \quad 98 \quad \mathrm{Kim}$, B. et al. Endothelial pyruvate kinase M2 maintains vascular integrity. J Clin Invest 648 128, 4543-4556, doi:10.1172/JCl120912 (2018).

$649 \quad 99$ Khan, S. et al. EndoDB: a database of endothelial cell transcriptomics data. Nucleic 650 Acids Res 47, D736-D744, doi:10.1093/nar/gky997 (2019). 651 


\section{FIGURE LEGENDS}

\section{FIGURE 1: ECS RELY ON METABOLIC REWIRING FOR ANGIOGENESIS}

654 Quiescent ECs adapt their metabolism to endure high oxygen levels, which they are exposed to. When 655 activated, ECs rewire their metabolism to sprout new vessels. Growth factors (schematically repre656 sented by green shapes) like fibroblast growth factor (FGF) or vascular endothelial growth factor 657 (VEGF) bind to receptors on ECs and induce proliferation of "stalk cells" and migration of "tip cells". 658 This phenotypic switch is dependent on metabolic changes in angiogenic ECs, which ensure amongst 659 other energy and biomass production for proliferation and migration.

\section{FIGURE 2: KEY METABOLIC PATHWAYS IN ECS.}

661 The functions of the major metabolic pathways in ECs are described in the main text. Proteins dis662 cussed in the main text are depicted in dark blue font, red symbols in the OXPHOS pathway represent 663 heme cofactors.

664 AbBReVIATIONS: 3PG, 3-phosphoglycerate; ACC, Acetyl-CoA Carboxylase; ASNS, Asparagine Synthetase; 665 ATP, Adenosine triphosphate; $\beta-O H B$, Beta hydroxybutyrate; CD36, CD36 molecule (fatty acid trans666 locase); CoA, Coenzyme A; CPT1A, Carnitine palmitoyltransferase 1A; DHODH, Dihydroorotate dehy667 drogenase; F2,6BP, Fructose-2,6-biphosphate; F6P, Fructose-6-phosphate; FAHD1, Fumarylacetoace668 tate hydrolase domain containing 1 (oxaloacetate decarboxylase); FASN, Fatty acid synthase; FATP, 669 Fatty acid transport protein; G3P, Glyceraldehyde-3-phosphate; G6P, Glucose-6-phosphate; G6PDH, 670 Glucose-6-phosphate dehydrogenase; GFAT1, Glutamine:fructose-6-phosphate amidotransferase 1; 671 GLS1, Glutaminase 1; GLS2, Glutaminase 2; GLUT1, Glucose transporter 1; GP, Glycogen phosphory672 lase; GS, Glutamine synthetase; GSH, Glutathione; HK2, Hexokinase 2; IDH2, Isocitrate dehydrogenase

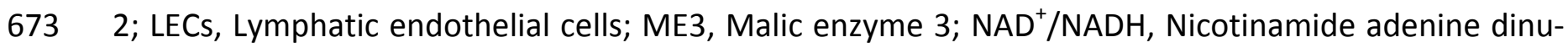


674 cleotide (oxidized/reduced); NADP ${ }^{+} / \mathrm{NADPH}$, Nicotinamide adenine dinucleotide phosphate (oxi675 dized/reduced); NFkB, nuclear factor kappa-light-chain-enhancer of activated B cells; OXCT1, 3676 oxoacid CoA-transferase 1; OXPHOS, Oxidative phosphorylation (mitochondrial respiration); PEP, 677 Phosphoenolpyruvate; PFKFB3, 6-phosphofructo-2-kinase/fructose-2,6-biphosphatase 3; PHGDH, 678 Phosphoglycerate dehydrogenase; PKM2, Pyruvate kinase isozyme M2; R5P, Ribose-5-phosphate; 679 SDH, Succinate dehydrogenase; TCA, Tricarboxylic acid; TKT, Transketolase; UDP-GIcNAc, Uridine di680 phosphate $\mathrm{N}$-acetylglucosamine.

FigURE 3: ADAPTATIONS OF METABOLIC PATHWAYS IN QUIESCENT VERSUS ANGIOGENIC ECS

682 Quiescent ECs (a) adapt their metabolism to maintain redox homeostasis to cope with their oxidative 683 stress-prone high oxygen environment (e.g. by upregulating the expression of vasculoprotective pro684 teins and regeneration of NADPH, as well as by limiting ROS production). Angiogenic ECs (b) rewire 685 their metabolic pathways for energy and biomass production essential for cell proliferation and mi686 gration. The pathways and the corresponding text boxes are highlighted with different colors (yellow, 687 glycolysis; brown, pentose phosphate pathway (PPP); dark green, fatty acid oxidation; light red, redox 688 metabolism; pink, OXPHOS; light green, fatty acid synthesis). The width of the highlighted pathway 689 corresponds to the flux through this pathway. For reasons of clarity, the metabolic pathways are 690 shown only schematically; for names of metabolites and metabolic enzymes, see Figure 2.

691 Abbreviations: ACC, Acetyl-CoA Carboxylase; AcCoA, Acetyl-CoA; Asp, Aspartate; ATP, Adenosine tri692 phosphate; $\mathrm{Cl}$, Mitochondrial complex I; CIII, Mitochondrial complex III; CPT1A, Carnitine palmito693 yltransferase 1A; DNA, Deoxyribonucleic acid; eNOS, Endothelial nitric oxide synthase 3; FA, Fatty ac694 id; FASN, Fatty acid synthase; FOXO1, Forkhead box protein 01; G6PDH, Glucose-6-phosphate dehy695 drogenase; GLRX2, Glutaredoxin 2; GPX3, glutathione peroxidase 3; HK2, Hexokinase 2; IDH2, Iso696 citrate dehydrogenase 2; ME3, Malic enzyme 3; NAD+/NADH, Nicotinamide adenine dinucleotide (ox- 
697 idized/reduced); NADP+/NADPH, Nicotinamide adenine dinucleotide phosphate (oxidized/reduced); 698 OXPHOS, Oxidative phosphorylation (mitochondrial respiration); PAH, Pulmonary arterial hyperten699 sion; PFKFB3, 6-phosphofructo-2-kinase/fructose-2,6-biphosphatase 3; PRDX1, Peroxiredoxin 1; PTM: 700 post-translational modification (malonylation; palmitoylation); ROS, Reactive oxygen species; TCA, 701 Tricarboxylic acid; TECs, Tumor endothelial cells.

\section{Figure 4: AdAPTATIONS OF METABOLIC PATHWAYS IN LYMPHATIC ECS}

703 When stimulated by growth factors, lymphatic ECs (LECs) undergo metabolic adaptations (i.e. upregu704 lation of ketone body oxidation (highlighted in red) and fatty acid oxidation (highlighted in dark 705 green)) to increase the synthesis of acetyl-CoA, which sustains the TCA cycle (in conjunction with ana706 plerotic substrates), resulting in the production of aspartate, a nucleotide precursor. The lymphatic 707 master regulator PROX1 increases FAO by upregulating Cpt1a gene expression that leads to increased 708 synthesis of acetyl-CoA, which is used for histone acetylation of PROX1 target genes by the histone 709 acetylase p300, thereby facilitating transcription of PROX1 targets and lymphatic differentiation of 710 ECs. For reasons of clarity, the metabolic pathways are shown only schematically; for names of me711 tabolites and metabolic enzymes, see Figure 2.

712 AbbreViations: Asp, Aspartate; ATP, Adenosine triphosphate; CPT1A Carnitine palmitoyltransferase 1A; 713 DNA, Deoxyribonucleic acid; OXCT1, 3-oxoacid CoA-transferase 1; p300, Histone acetyltransferase 714 p300; PROX1, Prospero Homeobox 1.

715 
718 Angiogenic ECs upregulate amino acid synthesizing pathways, which are used for protein and nucleo719 tide synthesis, redox homeostasis or posttranslational protein modification (PTM; palmitoylation). 720 Serine biosynthesis (Ser, highlighted in light blue) promotes nucleotide synthesis via one carbon me721 tabolism and synthesis of the antioxidant glutathione (GSH) and heme, a co-factor of OXPHOS com722 plexes, thereby securing mitochondrial respiration and dCTP synthesis by DHODH. Glutamine (Gln, 723 highlighted dark blue) is taken up from the environment and used to fuel the TCA cycle for biomass 724 production or asparagine synthesis (Asn). In contrast, glutamine synthase (GS) is negligible for gluta725 mine synthesis but regulates EC migration as palmitoyl transferase of RhoJ, a regulator of cytoskeletal 726 organization. For reasons of clarity, the metabolic pathways are shown only schematically; for names 727 of metabolites and metabolic enzymes, see Figure 2.

728 AbBreviations: ASNS, Asparagine Synthetase; ATP, Adenosine triphosphate; dATP, Deoxyadenosine 729 triphosphate; dCTP, Deoxycytidine triphosphate; dGTP, Deoxyguanosine triphosphate; DHODH, Dihy730 droorotate dehydrogenase; dTTP, Deoxythymidine triphosphate; GLS1, Glutaminase 1; Glu, Gluta731 mate; GS, Glutamine synthetase; GS, Glutamine synthetase; PHGDH, Phosphoglycerate dehydrogen732 ase; RhoJ, Ras homolog family member J; TCA, Tricarboxylic acid; TECs, Tumor endothelial cells.

\section{Figure 6: TheRAPEUtic StRATEgIES TARGETING EC METABOLISM}

734 A) In response to growth factors released by cancer cells, glycolysis in TECs is hyperactivated, leading 735 to hyperproliferation and impaired vascular barrier integrity, which compromises blood flow and facil736 itates cancer cell intravasation and metastasis (left panel). Inhibiting glycolysis moderately by approx737 imately $20 \%$ (using a low dose of the PFKFB3 inhibitor 3PO) normalizes the glycolytic flux to levels ob738 served in quiescent ECs, which reduces EC proliferation and re-establishes vessel barrier integrity. This 739 reduces metastasis and re-establishes flow in tumor blood vessels, which promotes delivery of anti- 
740 tumor drugs (middle panel). In contrast, glycolysis inhibition by $40 \%$ or more using a maximally toler741 able 3PO dose is toxic for ECs, leading to vessel disintegration and enhanced metastasis (right panel).

742 B) In patients with operable breast cancer, lymph nodes are often surgically removed. This disrupts 743 the lymphatic vessel network, which can lead to fluid accumulation and the development of 744 Iymphedema (left). Administration of ketone bodies via a ketogenic diet to the disrupted lymphatic 745 vessels stimulates the formation of new lymphatic vessels (lymphangiogenesis), which reduces 746 lymphedema (right).

747 Abbreviations: 3PO, Selective inhibitor of PFKFB3; ATP, Adenosine triphosphate; PFKFB3, 6748 phosphofructo-2-kinase/fructose-2,6-biphosphatase 3.

749

750 
Figure 1
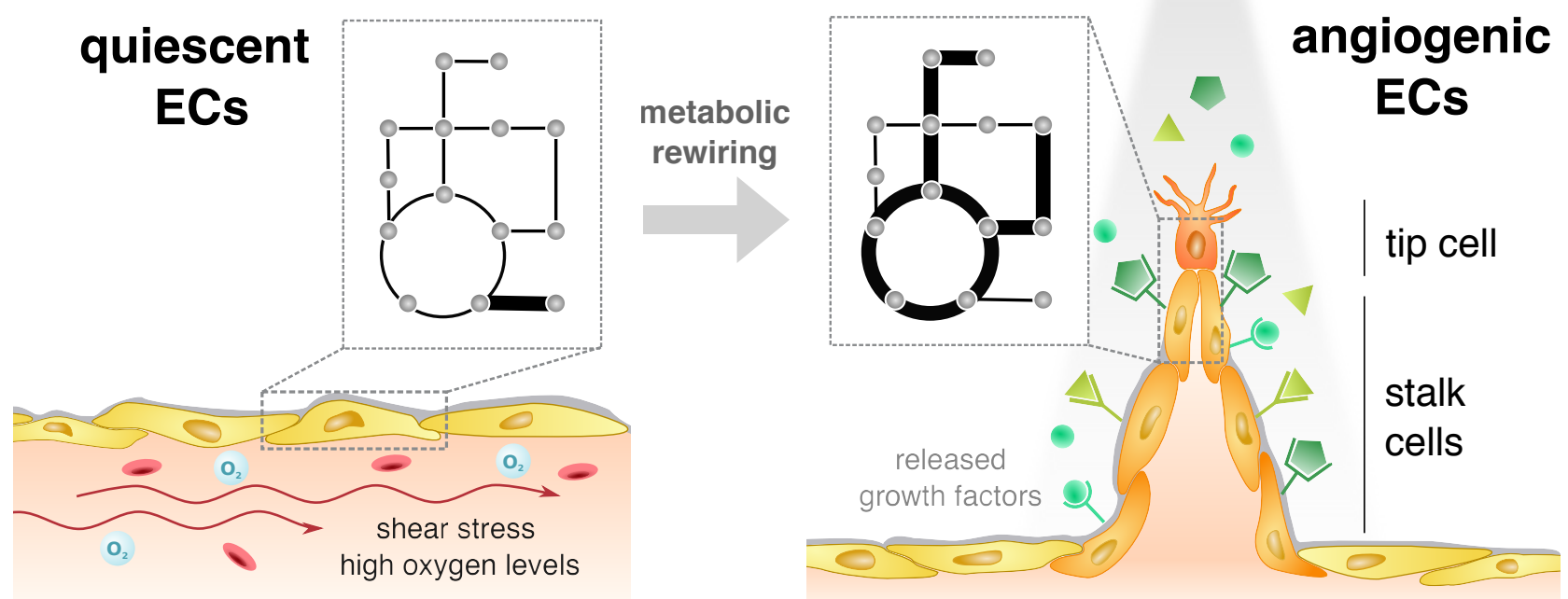
Figure 2

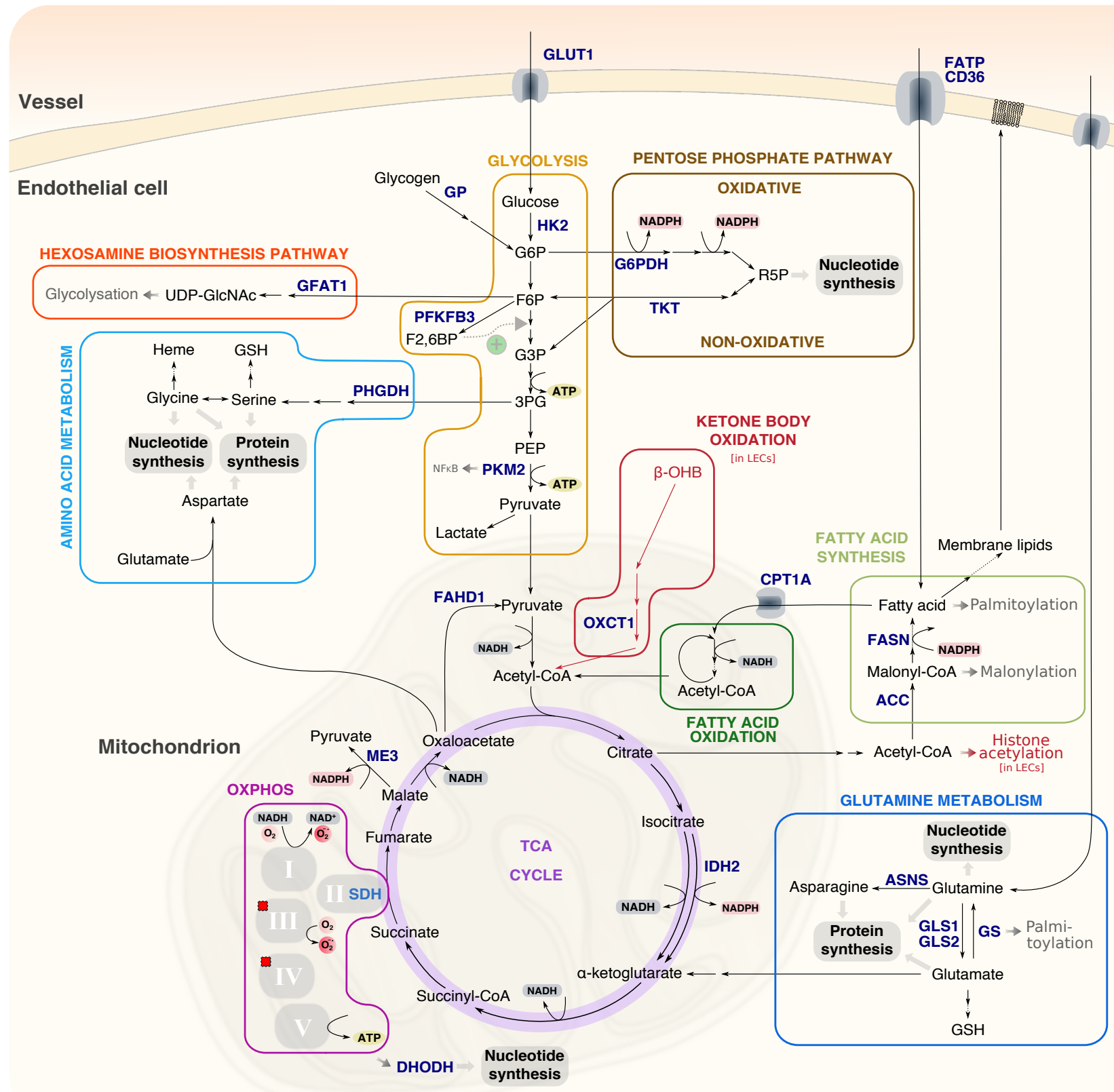


Figure 3

a

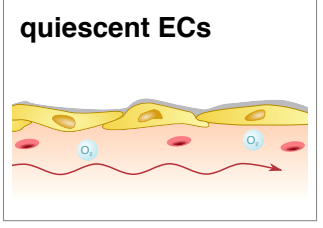
to oxPPP for redox homeostasis downregulated by $\mathrm{FOXO1}$ and $\mathrm{NOTCH}$

GLYCOLYSIS

energy production

carbon source

for TCA cycle

glycolytic intermediates

tasis
( $85 \%$ of generated ATP)
REDOX METABOLISM

vasculoprotection

upregulated redox homeostasis

(GLRX2, GPX3, PRDX1, eNOS)

and NADPH regeneration

(G6PDH, CPT1a, ME3, IDH2)

induced by Notch to protect against high oxygen environment

\section{ent}

b

\section{GLYCOLYSIS}

allows vascularization of $\mathrm{O}_{2}$ deprived tissues and supports motility of tip cells

high energy production

glycolytic intermediates to side pathways (PPP, serine synthesis) for biomass production

$\uparrow$ in TECs and PAH-ECs targets: PFKFB3, HK2

proliferation and migration

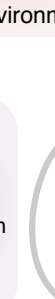

$\mathrm{H}$

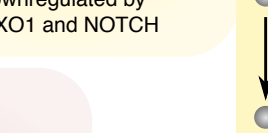

\section{。}

NADPH

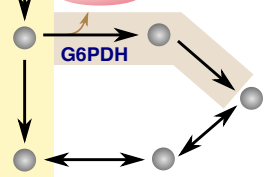

PPP

redox homeostasis

by NADPH regeneration

upregulated oxidative branch

\section{FA OXIDATION}

redox homeostasis through NADPH regeneration by IDH2 \& ME3

upregulated by FOXO1 and NOTCH

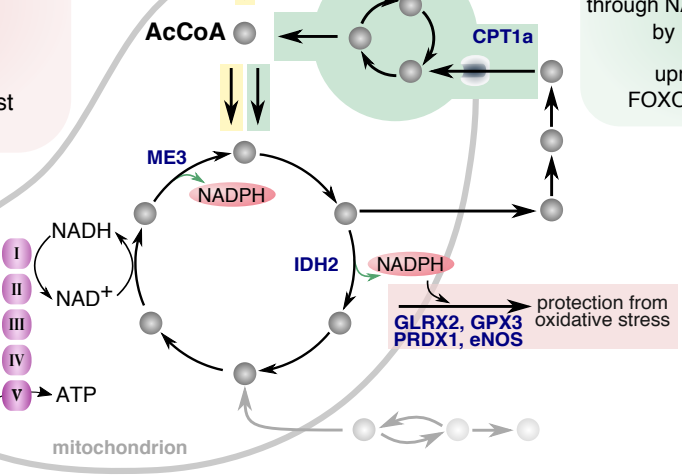

PPP

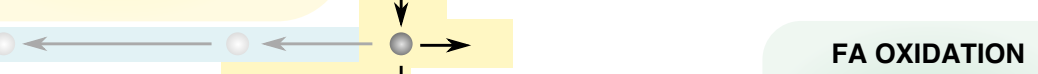

\section{SERINE} SYNTHESIS see Figure 5

\section{Protein synthesis}

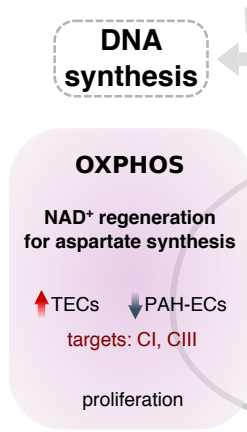

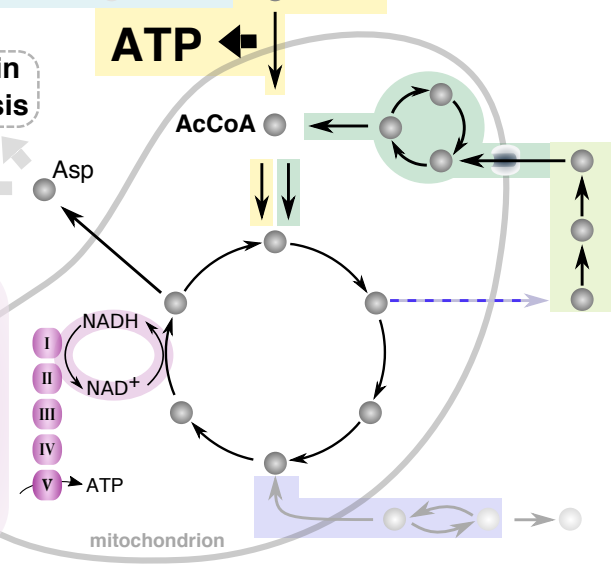

GLUTAMINE OXIDATION sustains TCA cycle to support

nucleotide synthesis

(without anaplerosis)

target: CPT1a

proliferation

\section{FA SYNTHESIS}

phospholipid composition

\& protein regulation

$\uparrow \mathrm{PAH}-\mathrm{ECS}$

targets: ACC, FASN

proliferation and/or migration 
Figure 4

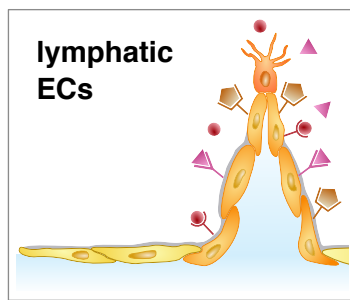

KETONE BODY

\section{METABOLISM}

sustains TCA cycle to support nucleotide synthesis

(without anaplerosis)

lymphangiogenesis

\section{FA OXIDATION}

histone acetylation

promoting expression

of lymphatic genes by PROX1

LEC differentiation

sustains TCA cycle to

support nucleotide synthesis

(without anaplerosis)

lymphangiogenesis

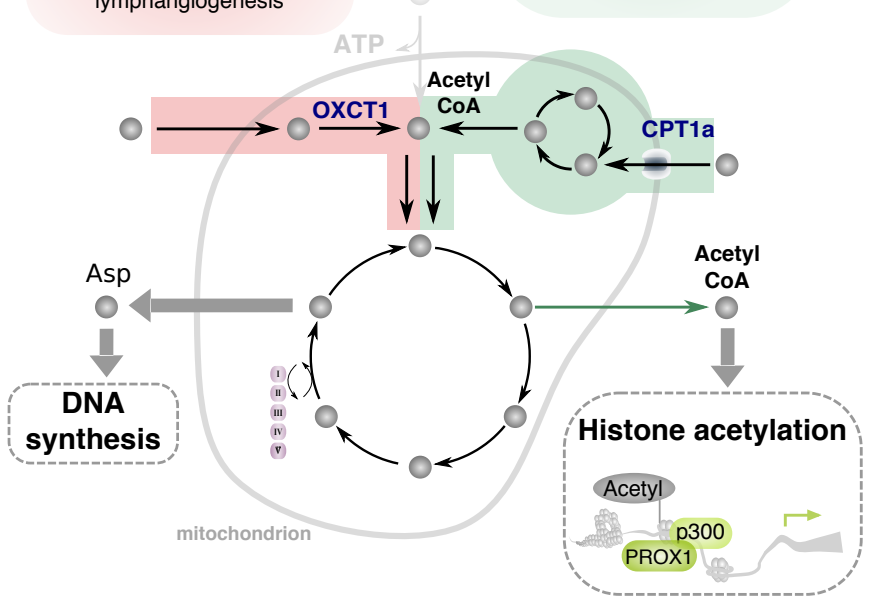


Figure 5
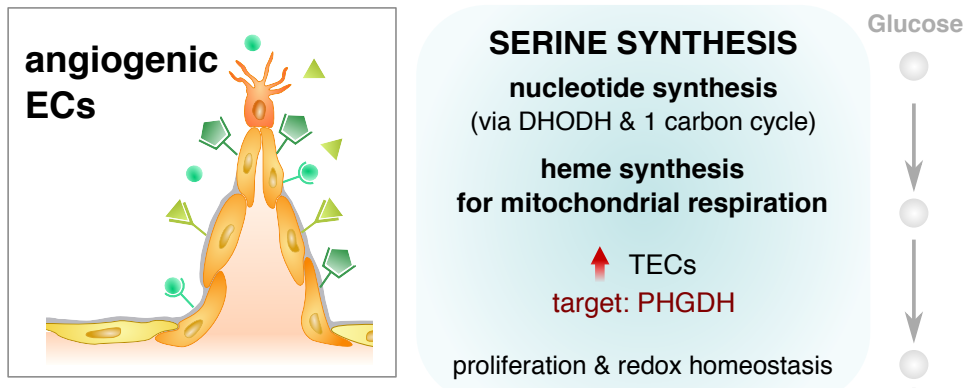

proliferation \& redox homeostasis

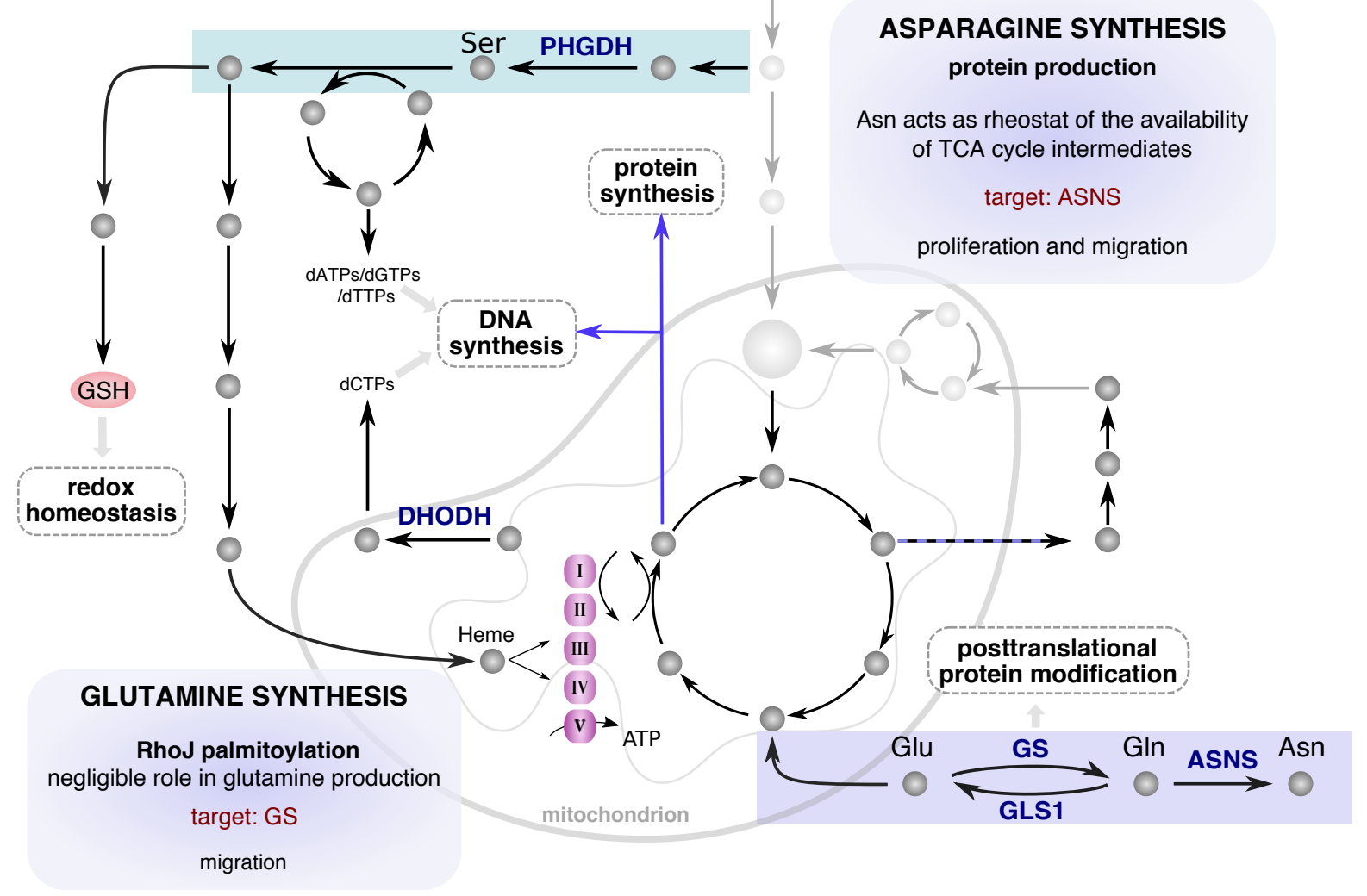

GLUTAMINE METABOLISM

nucleotide, amino acid

and lipid production,

redox homeostasis and others

- uptake in sprouting ECs

target: GLS1

proliferation and migration

\section{ASPARAGINE SYNTHESIS}

protein production

of TCA cycle intermediates

target: ASNS

proliferation and migration

posttranslationa

protein modification

Glu

migration 
Figure 6

a
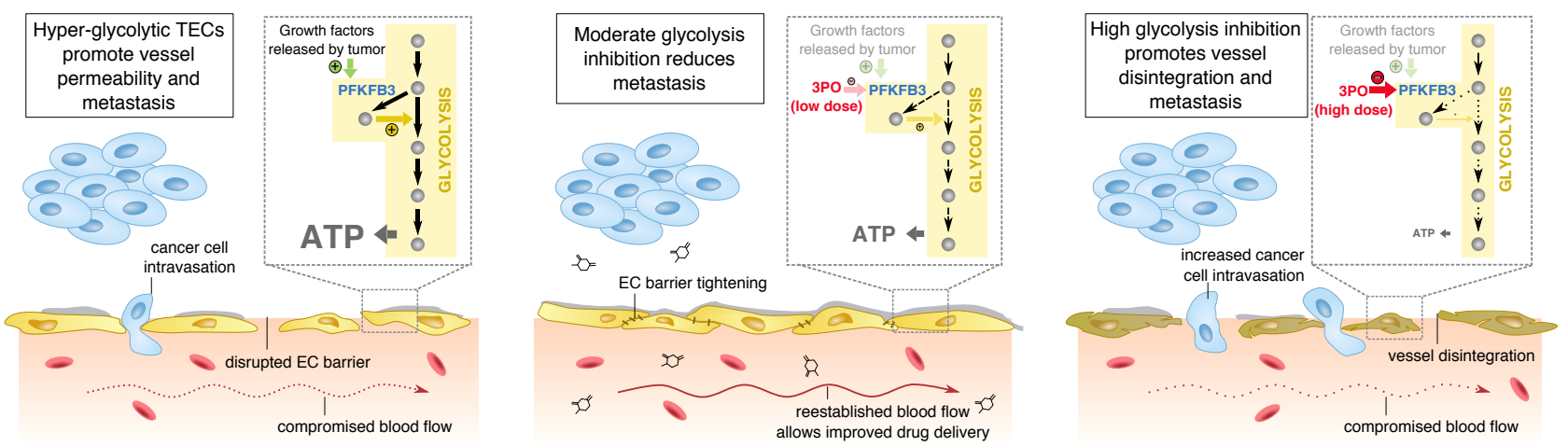

b
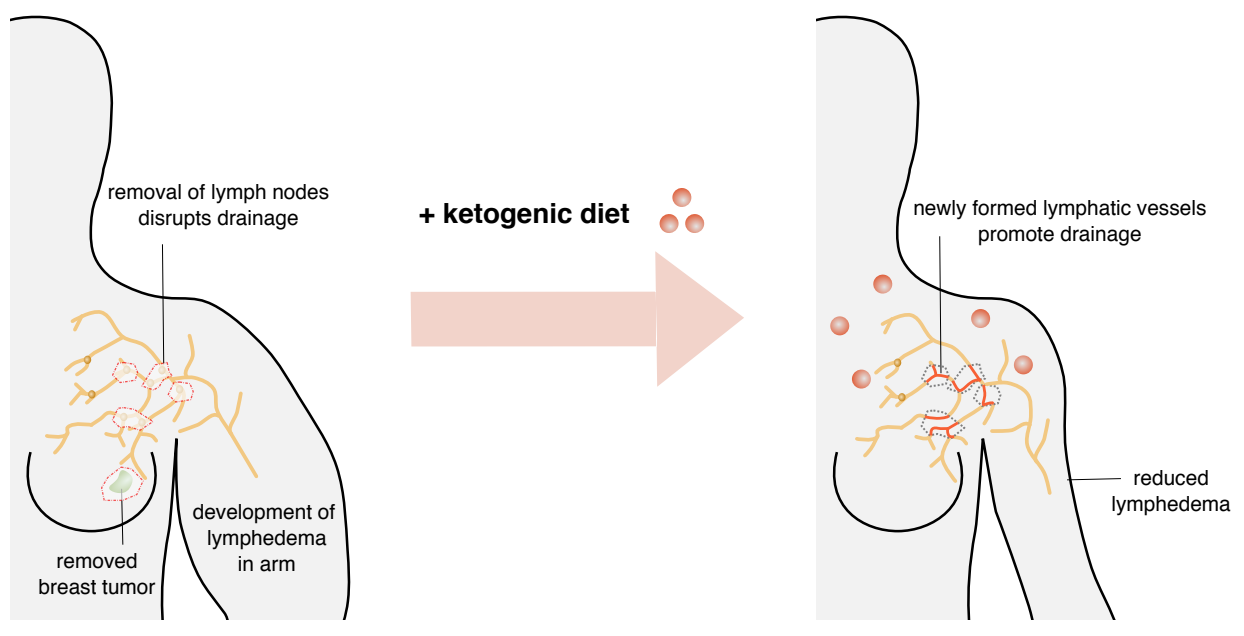